\title{
Algoritmo para redução de dados em redes de sensores baseado em Teoria da Informação
}

\author{
Givanildo L. N. Júnior ${ }^{1}$, Cristopher G. S. Freitas ${ }^{1}$, Osvaldo A. Rosso ${ }^{2}$, \\ André Luiz Lins Aquino ${ }^{1}$ \\ ${ }^{1}$ Instituto de Computação, Universidade Federal de Alagoas \\ ${ }^{2}$ Instituto de Física, Universidade Federal de Alagoas \\ givanildo.lima@laccan.ufal.br, cristopher@laccan.ufal.br \\ oarosso@gmail.com, alla.linslgmail.com
}

\begin{abstract}
This work proposes a data flow reduction algorithm based on the behavior of time series in the Complexity-Entropy plane for wireless sensor networks (WSNs). The system dynamic variation is identified in real time through a delimiter built into the plane, called the Maximum Complexity Cut-off Point. Thus, we can determine at which instants the sample interval must be updated in order to maximize the statistical complexity of the resulting data sample. This method was applied to a chaotic database and the obtained results were compared with those of other sampling algorithms, presenting better performance in the statistical metrics evaluated.
\end{abstract}

Resumo. Este trabalho propõe um algoritmo de redução do fluxo de dados baseado no comportamento de séries temporais no plano Complexidade-Entropia para redes de sensores sem fio (RSSF). A variação da dinâmica do sistema é identificada em tempo real através de um delimitador construído dentro do plano, denominado Ponto de Corte de Complexidade Máxima. Assim, podemos determinar em quais instantes se deve atualizar o intervalo de amostragem, de modo a maximizar a complexidade estatística da amostra de dados resultante. Este método foi aplicado a uma base de dados caóticos e os resultados obtidos foram comparados com os de outros algoritmos de amostragem, apresentando melhor desempenho nas métricas de estatística avaliadas.

\section{Introdução}

Uma rede de sensores sem fio, do inglês Wireless Sensor Network (WSN), é um tipo especial de rede ad hoc que tem capacidade de realizar detecção e processamento, podendo ser aplicada a muitos campos, como industrial, agrícola, ambiental e militar [Arampatzis et al. 2005]. Basicamente, essas redes são compostas por dispositivos compactos e autônomos distribuídos espacialmente e que atuam cooperativamente, chamados de nós sensores. Existem dois tipos principais de aplicações para redes de sensores: aplicações de monitoramento e acionamento. Nas aplicações de monitoramento, os nós somente processam os dados, enquanto que nas de acionamento, os nós podem interferir no ambiente que está sendo monitorado [Lins et al. 2003].

Em aplicações WSN, variáveis físicas, como temperatura e pressão, podem ser monitoradas continuamente ao longo do tempo de operação da rede. $\mathrm{O}$ conjunto de dados que representa essas variáveis pode ser referido como fluxo de dados 
[Muthukrishnan et al. 2005] ou fluxo do sensor. Entretanto, apesar do enorme potencial de aplicabilidade, uma rede de sensores possui uma série de restrições de recursos, como um baixo poder computacional, largura de banda reduzida e fonte de energia limitada.

Em algumas dessas aplicações, os dados chegam de forma online, são ilimitados e não há um ordenamento na chegada dos dados que serão processados. Então, em decorrência das limitações apresentadas por uma WSN que foram listadas acima, enviar uma grande quantidade de dados pode levar muito tempo quando os nós sensores competem para acessar o meio sem fio em uma comunicação de dados multihop. Portanto, faz-se necessário o uso de algoritmos que atuem sobre o fluxo de dados gerados a fim de reduzir o tráfego na rede.

Alguns exemplos de algoritmos que atuam na redução do fluxo do sensor, com variadas estratégias, são [Vasconcelos et al. 2015], que apresenta uma estratégia de descarte de pacotes com reconhecimento de dados, aplicando um algoritmo de amostragem nos dados coletados mantendo a qualidade geral dados para representar o fenômeno monitorado, [Jain and Chawla 2014], que apresentam algumas estratégias gerais para descarte de pacotes, como descartar o último pacote, descarte baseado no tamanho da mensagem, descarte baseado no número de encaminhamentos, dentre outras. Outras estratégias serão apresentadas posteriormente, na seção 3, a fim de comparar os resultados com os obtidos pela nossa abordagem.

Nesse sentido, este trabalho propõe um algoritmo de fluxo de dados para WSNs baseado em conceitos de teoria de informação. Esta solução estuda o comportamento da série temporal segundo as definições da entropia normalizada de Shannon $H$ e complexidade estatística $C$ no plano $H$ x $C$, ajustando dinamicamente o intervalo de amostra de acordo com a detecção da variação do comportamento do sistema, utilizando limites estabelecidos pelo Ponto de Corte de Complexidade Máxima. Nessa abordagem, o intervalo de amostragem é calculado e atualizado em tempo real, permitindo que este algoritmo seja utilizado em aplicações WSN online. Para aplicabilidade deste trabalho, foram utilizadas 27 séries temporais produzidas a partir de mapas caóticos. Usando a solução de amostragem proposta, foi possível reduzir o tráfego de dados, preservando as propriedades gerais da série $\mathbf{X}$ na amostra resultante.

As demais seções deste trabalho organizam-se em: Seção 2 que está subdivida em Seção 2.1, que apresenta os mapas caóticos utilizados, Seção 2.2, na qual são discutidos os quantificadores de Teoria da Informação e sua metodologia de utilização e Seção 2.3, que descreve a abordagem desenvolvida pelo algoritmo. Na Seção 3 são discutidos e avaliados os resultados obtidos, comparando-os com os de outros algoritmos de amostragem com abordagens distintas, e a Seção 4 encerra com as devidas conclusões.

\section{Materiais e Métodos}

\subsection{Mapas Caóticos}

Uma enorme variedade de sistemas apresenta comportamentos irregulares, em maior ou menor grau, como por exemplo os que descrevem o clima, certas reações químicas, tráfego de carros e alguns circuitos elétricos.

Desse modo, as não-linearidades presentes, que são comuns em quase todo sistema, tornam seu comportamento não trivial. Em particular, o caos é um dos compor- 
tamentos que pode ser observado em alguns sistemas não-lineares. Nesses casos, uma pequena perturbação nas condições iniciais pode resultar numa grande diferença em tempos posteriores, efeito denominado "sensibilidade às condições iniciais", que caracteriza o comportamento caótico de alguns sistemas não-lineares tornando-os imprevisíveis, de forma que trajetórias inicialmente muito próximas passam a divergir exponencialmente com o tempo.

Na década de 90, surgiram novos modelos de sistemas de comunicação [Hayes et al. 1993] que passaram a utilizar essas propriedades para transmissão de informações de modo eficiente. Desde então, vários estudos foram desenvolvidos utilizando sistemas caóticos em processos como compressão de dados [Masmoudi and Puech 2014], criptografia [Kocarev et al. 2005] e modulação [Eisencraft et al. 2018].

Em nossa abordagem, foram utilizados 27 mapas caóticos com suas equações geradoras descritas por Sprott no apêndice de seu livro [Sprott and Sprott 2003]. Para utilizar cada um deles, tomamos as mesmas condições iniciais e os parâmetros que foram detalhados por Sprott. Esses mapas caóticos são agrupados como segue:

- Mapas não inversíveis: (1) logistic map; (2) sine map ; (3) tent map; (4) linear congruential generator; (5) cubic map; (6) Ricker's population model; (7) Gauss map; (8) Cusp map; (9) Pinchers map; (10) Spence map; (11) sine-circle map

- Mapas dissipativos: (12) Hénon map; (13) Lozi map; (14) delayed logistic map; (15) Tinkerbell map; (16) Burgers' map; (17) Holmes cubic map; (18) dissipative standard map; (19) Ikeda map; (20) Sinai map; (21) discrete predator-prey map.

- Mapas conservativos: (22) Chirikov standard map; (23) Hénon area-preserving quadratic map; (24) Arnold's cat map; (25) Gingerbreadman map; (26) chaotic web map; (27) Lorenz three dimensional chaotic map.

\subsection{Teoria da Informação}

A entropia da série temporal é calculada utilizando o conceito da entropia clássica de Shannon para distribuições discretas. Dada uma função de probabilidade $P=\left\{p_{k}: k=\right.$ $1, \ldots, N\}$ sobre $N$ valores, essa medida é dada por:

$$
S(P)=-\sum_{k}^{N} p_{\kappa} \log p_{\kappa}
$$

A entropia clássica de Shannon [Shannon 1948] mede a desordem de um sistema, considerando a probabilidade desse sistema apresentar um estado $k$. Esse medida está relacionada com a informação associada ao processo físico descrito pela função de probabilidade $P$. Normalizando essa medida $\left(H_{s} \in[0,1]\right)$, temos

$$
H_{s}[P]=S[P] / S_{\max }
$$

onde $S_{\max }=S\left[P_{e}\right]=\ln N$ e $P_{e}=\{1 / N, \ldots, 1 / N\}$ é a distribuição uniforme.

[Lamberti et al. 2004] desenvolveram uma medida eficaz de complexidade estatística $\mathbf{C}$, que é capaz de: (i) Detectar detalhes essenciais da dinâmica e (ii) diferenciar 
entre o caos e periodicidade (em diferentes graus). Este medida de complexidade fornece algumas informações adicionais importantes à respeito da função de distribuição de probabilidade que não necessariamente são detectados pela entropia.

Então, seguindo a noção de complexidade apresentada em [Lopez-Ruiz et al. 1995], temos que a complexidade é dada pelo produto

$$
C_{J S}[P]=Q_{J}\left[P, P_{e}\right] H_{s}[P]
$$

sendo o desequilíbrio $Q_{J}$ medido através do divergente de Jensen-Shannon, que quantifica a diferença entre duas (ou mais) distribuições de probabilidade, dado por

$$
Q_{J}=Q_{0} J\left[P, P_{e}\right]
$$

com

$$
J\left[P, P_{e}\right]=S\left[\left(P+P_{e}\right) / 2\right]-S[P] / 2-S\left[P_{e}\right] / 2
$$

onde $Q_{0}$ é uma constante de normalização $\left(0 \leq Q_{J S} \leq 1\right)$ igual ao inverso do valor máximo possível de $J\left[P, P_{e}\right]$. Então, $Q_{0}$ é dado por:

$$
Q_{0}=-2 \cdot\left\{\left(\frac{N+1}{N}\right) \cdot \log (N+1)-2 \cdot \log (2 N)+\log (N)\right\}^{-1}
$$

A complexidade estatística de um sistema é nula em duas situações opostas: no conhecimento perfeito ou na aleatoriedade completa. Qualquer tipo de sistema se situará entre essas configurações extremas.

Nessa abordagem, dada uma série temporal $\mathbf{X}(t)=\left\{x_{t}: t=1, \ldots, N\right\}$, que representa o fenômeno avaliado, construímos a distribuição de probabilidade $P$ correspondente a distribuição proposta por [Bandt and Pompe 2002]. Para essa distribuição, computamos padrões ordinais de ordem $D$, para $D>1$, tal que

$$
(s) \mapsto\left(x_{s-(D-1)}, x_{s-(D-2)}, \ldots, x_{s-1}, x_{s}\right),
$$

onde para cada instante de tempo, temos um vetor de dimensão $D$ definido como $(s)$, constituindo uma janela deslizante com salto unitário, e gerando $\mathcal{Y}$ resultados, tal que $\mathcal{Y}=N-D+1$. Os padrões ordinais de cada vetor $(s)$ correspondem a uma permutação $\pi=\left\{r_{0}, r_{1}, \ldots, r_{D-1}\right\}$ de $\{0,1, \ldots, D-1\}$, tal que $x_{s-r_{D-1}} \leq x_{s-r_{D-2}} \leq \ldots \leq$ $x_{s-r_{1}} \leq x_{s-r_{0}}$. Dessa forma, nós convertemos o vetor $(s)$ para um símbolo $\pi$. Para garantir um resultado único, $r_{i}<r_{i-1}$ se $x_{s-r_{i}}=x_{s-r_{i-1}}$. Assim, para todas as $D$ ! permutações possíveis $\pi$ de ordem $D$, a distribuição de probabilidade $P \equiv\{p(\pi)\}$, é definida por

$$
p(\pi)=\frac{\#\{(s) \mid s \leq \mathcal{Y} ; \quad(s) \text { tem tipo } \pi\}}{\mathcal{Y}},
$$

sendo \# um operador de cardinalidade do conjunto. Em outras palavras, para qualquer série temporal, procuramos padrões ordinais de ordem $D$, que determina o número de 
estados acessíveis $D$ ! e a partir da frequência de ocorrência do símbolo, encontramos a distribuição de probabilidade de permutação.

Com isso, [Rosso et al. 2007] definiu o plano de causalidade ComplexidadeEntropia, utilizando a distribuição de probabilidade Bandt-Pompe com o objetivo de categorizar diferentes cenários de caos. Isso porque a complexidade é capaz de perceber detalhes da distribuição de probabilidade do sistema que não são discriminados por medidas de aleatoriedade, como a entropia [Feldman et al. 2008].

\subsection{Experimento}

Com o propósito de construir uma base de dados caóticos para análise, foram utilizados 27 diferentes mapas caóticos. A partir de cada um deles foram geradas sequências pseudoaleatórias de $10^{6}$ números. Em seguida, todos esses dados foram concatenados de modo a formar uma série temporal $\mathbf{X}$. Para determinar a melhor forma de amostrar esse conjunto de dados, foi utilizada uma janela de cobertura dos dados com comportamento semelhante ao de uma janela deslizante:

A janela possui um tamanho variável $T$, sendo este diretamente proporcional ao intervalo de amostragem utilizado $I$, tal que $T=1.800$.I. Dessa forma, independente do tamanho do janela, a quantidade de dados utilizada para determinar entropia $H$ e complexidade estatística $C$ da série temporal no intervalo é sempre a mesma, um conjunto de 1.800 valores. A escolha do valor 1.800 se deu de forma empírica, por representar um total de 30min em uma WSN que processa dados a cada segundo. A janela se desloca sempre dando um "salto"de 100 unidades, de modo a reavaliar dados já cobertos por janelas anteriores. Ao conjunto de dados amostrados a partir deste método damos o nome de $\mathbf{X}^{\prime}$.

$\mathrm{O}$ processo de amostragem é feito a partir do plano $H \times C$ utilizando um delimitador denominado Ponto de Corte de Complexidade Máxima. O plano de causalidade complexidade-entropia é definido como o diagrama bidimensional gerado quando se situa a complexidade estatística da permutação (eixo das ordenadas) versus a entropia de permutação (eixo das abscissas) para um determinado sistema [Rosso et al. 2007].

Inicialmente, utilizando um bloco com os 1.800 primeiros valores da base, são calculados entropia e complexidade, a fim de determinar o comportamento inicial da base de dados e dessa forma, construir o Ponto de Corte, o qual é determinado a partir de intervalos de estabilidade do sistema nos eixos de Entropia e Complexidade. Nesse caso, como todos os 1.800 valores são utilizados para cálculo, considera-se que o intervalo de amostragem foi de 1 segundo, o que significa que nenhum dado deve ser desprezado. Os valores calculados determinam um ponto no plano $H \times C$, que é o centro do Ponto de Corte. Os intervalos de estabilidade são definidos distando $10 \%$ do valor central em seu respectivo eixo, ou seja, os limites superior e inferior são $10 \%$ maior e $10 \%$ menor que o valor calculado da complexidade, respectivamente. De modo equivalente são definidos os limites esquerdo e direito, mas desta vez o valor central é o da entropia.

O próximo passo é utilizar os dados amostrados que estão englobados pela janela para cálculo de um novo par $(h, c)$. Então, é verificado se esse par está dentro dos limites determinados pelo Ponto de Corte. Em caso positivo, o intervalo de amostragem é dobrado, pois considera-se que o comportamento da Série Temporal X não sofreu nenhuma variação brusca, ou seja, se manteve dentro dos limites esperados. Entretanto, se o 
par estiver fora, um novo Ponto de Corte é construído centrado nesse par e o intervalo de amostragem volta a ser de apenas 1 segundo, ou seja, nenhum dado coletado é descartado. Desta forma, o processo de amostragem dos dados é feito em tempo real e vai se adaptando de acordo com o comportamento da série avaliada. Para encaixar o algoritmo em uma WSN, ele deve ser alocado em cada um dos sensores, para que cada um possa variar o intervalo de amostragem baseado nos dados capturados em seu respectivo ambiente.

\section{Resultados e Discussão}

Utilizando a abordagem descrita na seção anterior, a série $\mathbf{X}$, obtida utilizando os mapas caóticos, foi amostrada baseando-se no comportamento de sua complexidade estatística no plano $H \times C$, apresentado na figura 1. Como resultado, foi obtida uma série amostrada $\mathbf{X}^{\prime}$, com redução de $98,91 \%$ do tamanho original.

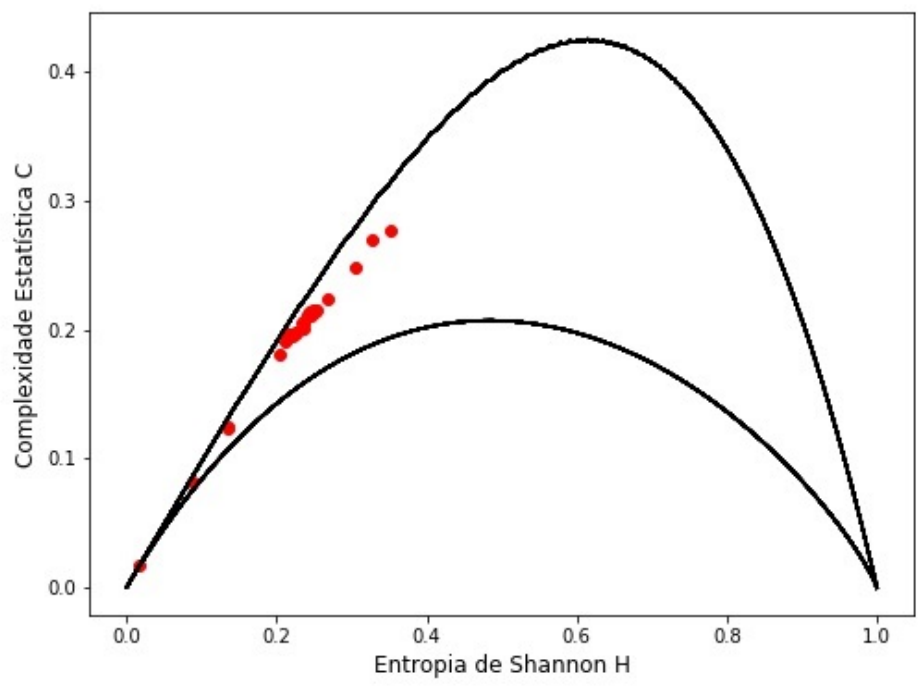

Figura 1. Comportamento apresentado pela série $X$ no Plano ComplexidadeEntropia durante o processo de amostragem

Com o propósito de verificar a consistência do método em sua capacidade de amostrar dados enquanto a complexidade do sistema aumenta, outros dois algoritmos de amostragem foram utilizados para fins de comparação:

- Em [de Aquino et al. 2007], o algoritmo proposto é baseado em técnicas de amostragem aplicadas a histogramas de dados criados a partir de fluxos de dados originais adquiridos por nós sensores. Como resultado, o algoritmo fornece uma amostra de apenas $\log (n)$ itens para representar os dados originais de $\mathrm{n}$ elementos.

- Em [Aquino et al. 2010], o algoritmo de amostragem é baseado em transformadas wavelet com base em Coiflets para reduzir o montante de dados coletados em aplicações de redes de sensores sem fio. A base dos Coiflets é mais computacionalmente eficiente quando os dados são suaves, o que significa que os dados são bem aproximados por uma função polinomial. A principal contribuição deste algoritmo foi sua capacidade de detectar algum evento, ajustando a amostragem dinamicamente. 
Nessa etapa, os outros 2 algoritmos de amostragem foram aplicados à série $\mathbf{X}$ modificando os devidos parâmetros para que o percentual de redução obtido fosse o mais próximo possível dos $98,91 \%$ obtidos com a abordagem do plano $H$ x $C$.

Quando aplicamos algoritmos de amostragem sobre fluxos de dados, a qualidade desses dados diminui. Logo, para atender aos requisitos da aplicação, devemos analisar esse impacto. O erro entre o fluxo original e o amostrado pode estar relacionado à distribuição e à discrepância de seus valores.

Para avaliar a aproximação da distribuição entre o conjunto de dados original e o amostrado, foi aplicado o teste Kolmogorov-Smirnov. O teste Kolmogorov-Smirnov (também conhecido como teste K-S) é um teste não paramétrico acerca da igualdade entre distribuições de probabilidade contínuas e unidimensionais que pode ser usado para comparar duas amostras entre si (chamado de teste K-S biamostral) [Daniel et al. 1978]. $\mathrm{O}$ teste $\mathrm{K}-\mathrm{S}$ biamostral é uma das técnicas não paramétricos mais úteis e conhecidas para comparar duas amostras, pois é sensível a diferenças tanto no local, como na forma das funções das duas amostras [Corder and Foreman 2009]. Os resultados obtidos são apresentados na figura 2 e mostram que nosso algoritmo teve distância vertical inferior aproximadamente 0.076 a [de Aquino et al. 2007] e 0.307 a [Aquino et al. 2010].

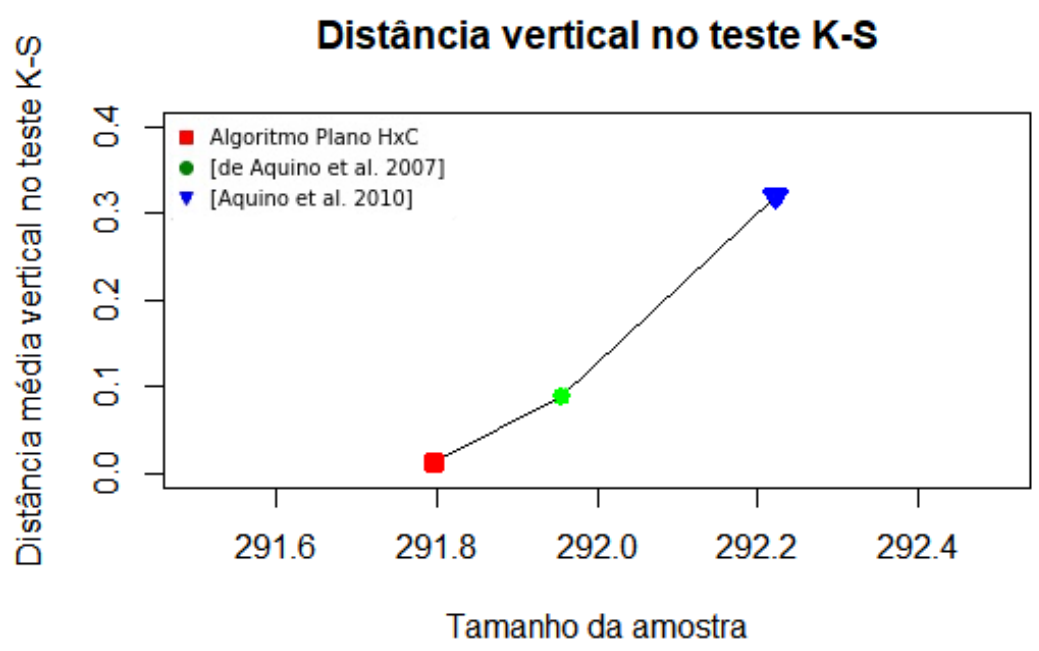

Figura 2. Comparação das das distâncias médias verticais obtidas por cada algoritmo utilizando o teste K-S

Além disso, como o teste K-S quantifica apenas se as distribuições são semelhantes, também é importante avaliar a discrepância dos valores nas amostras, ou seja, se elas são representativas da série original. Para quantificar essa discrepância, calculamos o valor absoluto da maior distância entre a média dos dados originais e os valores do intervalo de confiança de 95\%, inferior e superior, da média dos dados amostrados. Dessa forma, temos o chamado Erro de Dados que é definido como Erro de dados $=\operatorname{Max}\left\{\mid\right.$ inferior $_{\text {amostra }}-$ media $_{\text {original }}|$,$| superior _{\text {amostra }}-$ media $\left._{\text {original }} \mid\right\}$, onde

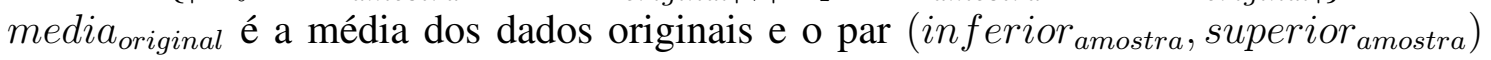
é o intervalo de confiança da amostra. Os resultados calculados do Erro de dados são exibidos na figura 3 e mostram que nosso algoritmo apresentou erro médio inferior 
apenas 0.42 a [de Aquino et al. 2007], enquanto a diferença para [Aquino et al. 2010] foi de aproximadamente 107.2.

\section{Erro de dados}

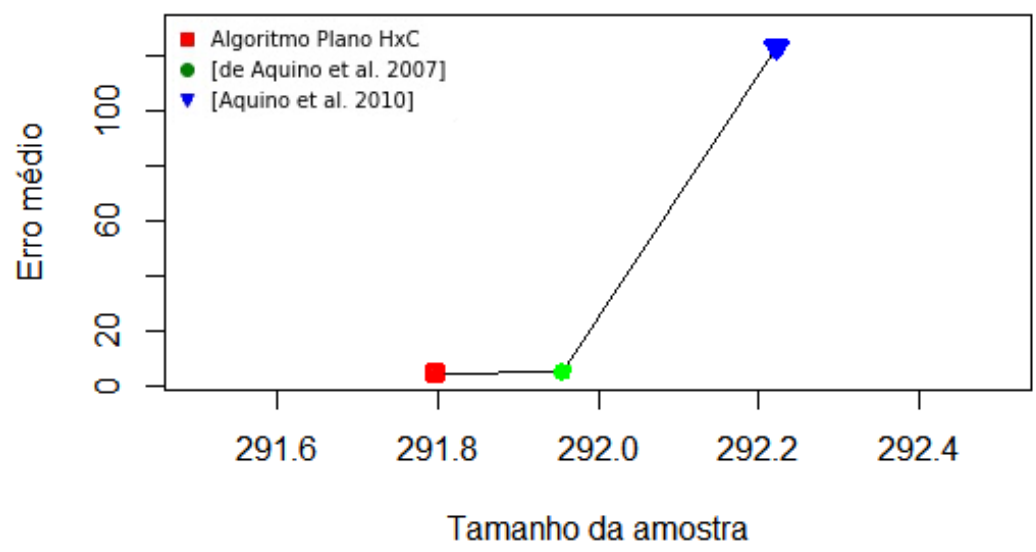

Figura 3. Comparação do erro de dados de cada algoritmo

Em ambas as métricas avaliadas, nosso método obteve resultados melhores, indicando que a amostra resultante dele é a mais representativa da série $\mathbf{X}$ dentre as analisadas.

Em seguida, foram calculadas a complexidade e entropia de cada amostra e os resultados foram plotados no plano $H \times C$. Como mostra a figura 4, a complexidade da amostra obtida utilizando teoria da informação é superior as demais, o que indica que há uma quantidade superior de informações relevantes na série resultante.

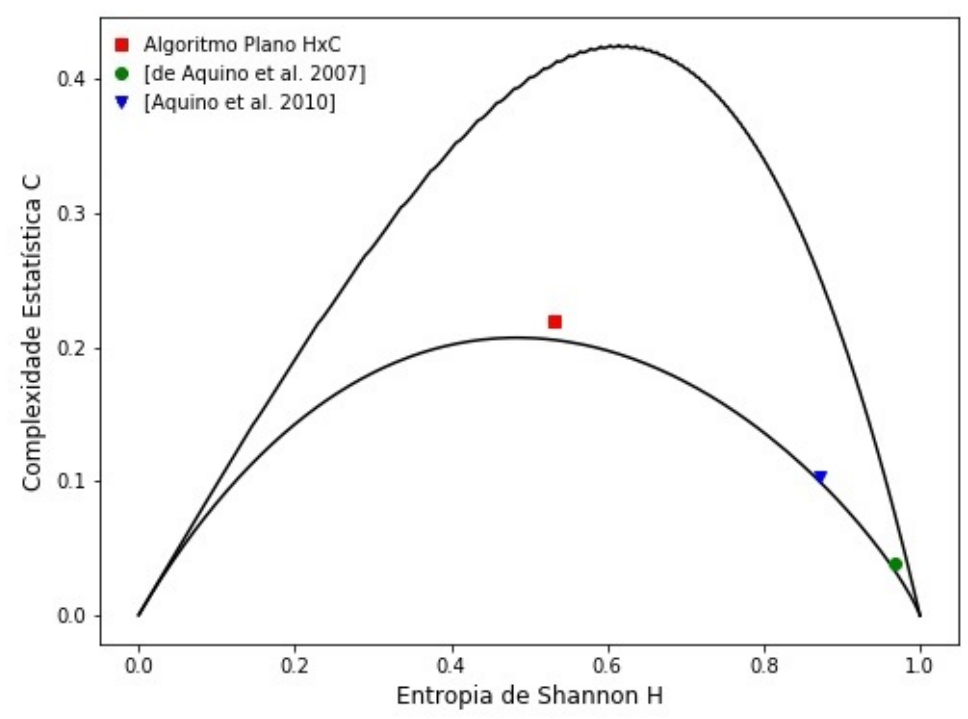

Figura 4. Comparação das complexidades das amostras referentes a cada algoritmo 


\section{Conclusão}

Em aplicações que utilizam redes de sensores sem fio (WSNs), existe uma série de restrições de recursos, como fonte de energia limitada, baixo poder computacional e uma largura de banda reduzida. Em função desses problemas, é necessário utilizar estratégias que atuem sobre o fluxo de dados gerados com a finalidade de reduzir o tráfego na rede.

Neste trabalho, foi apresentado um algoritmo de amostragem de dados em tempo real para WSNs. A ideia principal do algoritmo de amostragem é manter os dados mais relevantes para representar os fenômenos observados, descartando os mais redundantes e elevando a complexidade estatística da amostra. Para isso, o algoritmo se baseia no comportamento apresentado pela série temporal no plano Complexidade-Entropia. Utilizando os próprios dados processados, é construído um delimitador denominado Ponto de Corte de Complexidade Máxima, utilizado para identificar variações na dinâmica do sistema em tempo real e desse modo, determinar em quais momentos o intervalo de amostragem deve ser atualizado.

A pricipal contribuição deste trabalho foi apresentar um algoritmo eficaz para redução do fluxo de dados em uma WSN, mantendo a representatividade da série original, como mostram os resultados dos testes aplicados. Além disso, vale destacar que os resultados da amostragem utilizando o plano Complexidade-Entropia foram comparados com os resultados obtidos a partir de outras duas abordagens distintas, tendo desempenho superior em todas as métricas avaliadas. No teste KS, este algoritmo apresentou distância vertical inferior aproximadamente 0.076 a [de Aquino et al. 2007] e 0.307 a [Aquino et al. 2010], enquanto o erro médio foi inferior apenas 0.42 a [de Aquino et al. 2007] e aproximadamente 107.2 para [Aquino et al. 2010].

Em nossos trabalhos futuros, pretendemos avaliar o impacto de nossa abordagem sobre o consumo de energia e o atraso de pacotes em uma WSN. Além disso, pretendemos utilizar outras métricas para analisar o comportamento do nosso algoritmo, comparando com outros algoritmos de amostragem existentes.

\section{Referências}

Aquino, A. L., Oliveira, R. A., and Wanner, E. F. (2010). A wavelet-based sampling algorithm for wireless sensor networks applications. In Proceedings of the 2010 ACM Symposium on Applied Computing, pages 1604-1608. ACM.

Arampatzis, T., Lygeros, J., and Manesis, S. (2005). A survey of applications of wireless sensors and wireless sensor networks. In Proceedings of the 2005 IEEE International Symposium on, Mediterrean Conference on Control and Automation Intelligent Control, 2005., pages 719-724. IEEE.

Bandt, C. and Pompe, B. (2002). Permutation entropy: a natural complexity measure for time series. Physical review letters, 88(17):174102.

Corder, G. W. and Foreman, D. I. (2009). Nonparametric statistics for non-statisticians: a step-by-step approach. John Wiley \& Sons.

Daniel, W. W. et al. (1978). Applied nonparametric statistics. Houghton Mifflin.

de Aquino, A. L., Figueiredo, C. M. S., Nakamura, E. F., Buriol, L. S., Loureiro, A. A. F., Fernandes, A. O., and Coelho Jr, C. J. N. (2007). A sampling data stream algorithm for 
wireless sensor networks. In 2007 IEEE International Conference on Communications, pages 3207-3212. IEEE.

Eisencraft, M., Attux, R., and Suyama, R. (2018). Chaotic signals in digital communications. CRC Press.

Feldman, D. P., McTague, C. S., and Crutchfield, J. P. (2008). The organization of intrinsic computation: Complexity-entropy diagrams and the diversity of natural information processing. Chaos: An Interdisciplinary Journal of Nonlinear Science, 18(4):043106.

Hayes, S., Grebogi, C., and Ott, E. (1993). Communicating with chaos. Physical review letters, 70(20):3031.

Jain, S. and Chawla, M. (2014). Survey of buffer management policies for delay tolerant networks. The Journal of Engineering, 2014(3):117-123.

Kocarev, L., Makraduli, J., and Amato, P. (2005). Public-key encryption based on chebyshev polynomials. Circuits, Systems and Signal Processing, 24(5):497-517.

Lamberti, P., Martin, M., Plastino, A., and Rosso, O. (2004). Intensive entropic nontriviality measure. Physica A: Statistical Mechanics and its Applications, 334(12):119-131.

Lins, A., Nakamura, E. F., Loureiro, A. A., and Coelho, C. J. (2003). Beanwatcher: a tool to generate multimedia monitoring applications for wireless sensor networks. In IFIP/IEEE International Conference on Management of Multimedia Networks and Services, pages 128-141. Springer.

Lopez-Ruiz, R., Mancini, H. L., and Calbet, X. (1995). A statistical measure of complexity. Physics Letters A, 209(5-6):321-326.

Masmoudi, A. and Puech, W. (2014). Lossless chaos-based crypto-compression scheme for image protection. IET Image Processing, 8(12):671-686.

Muthukrishnan, S. et al. (2005). Data streams: Algorithms and applications. Foundations and Trends $\mathrm{R}$ in Theoretical Computer Science, 1(2):117-236.

Rosso, O., Larrondo, H., Martin, M., Plastino, A., and Fuentes, M. (2007). Distinguishing noise from chaos. Physical review letters, 99(15):154102.

Shannon, C. E. (1948). A mathematical theory of communication. Bell system technical journal, 27(3):379-423.

Sprott, J. C. and Sprott, J. C. (2003). Chaos and time-series analysis, volume 69. Citeseer.

Vasconcelos, I. L., Lima, D. H., Figueiredo, C. M., and Aquino, A. L. (2015). A sampling algorithm for intermittently connected delay tolerant wireless sensor networks. In 2015 IEEE Symposium on Computers and Communication (ISCC), pages 538-543. IEEE. 